\section{Helicobacter pylori hideout in gastric glands}

Microniches within the gastric glands of the stomach house a reservoir of Helicobacter pylori, according to new research in mice. The findings indicate a potential strategy by which $H$. pylori bacteria colonize and persist in the stomach.

Mice were infected with $H$. pylori PMSS1 strains that expressed different coloured fluorescent proteins to track the dynamics of mixed or sequential chronic infections in the stomach. These bacterial populations were visualized in the intact stomach using $3 \mathrm{D}$ confocal microscopy in combination with the passive CLARITY technique, which renders tissue optically transparent. "These high-resolution imaging methods allowed us to map the location of $H$. pylori through an entire organ with unprecedented spatial resolution," explains author Manuel Amieva.

The researchers observed that a small number of bacteria establish colonies deep in the gastric glands, with these populations able to expand in size and colonize adjacent glands over time, persisting throughout chronic infection. Notably, glandassociated populations did not mix with free-swimming bacteria in the surface mucus and excluded competitors from this microniche.

Host factors that regulate H. pylori gland colonization were also examined. Both the age at infection (mice infected as neonates had higher numbers of bacteria per gland than mice infected as adults) and host $\mathrm{T}$ cell responses affected the density of these gland-associated bacteria during chronic infection.

"Our results led us to propose that microniches in the gastric glands provide a protected niche for H. pylori to maintain a stable reservoir that can replenish the more transient populations in the superficial mucosa," says Amieva. "Given that

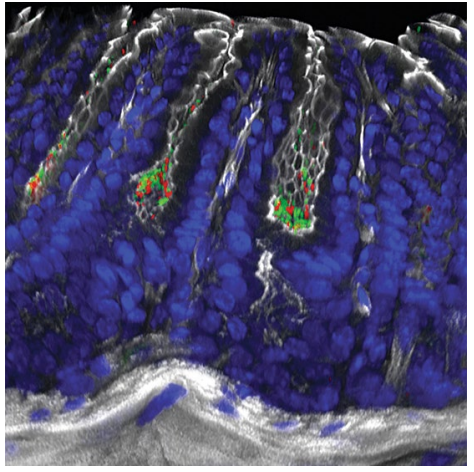

3D confocal image of mouse stomach infected with $\mathrm{H}$. pylori showing microcolonies in the gastric glands. Image courtesy of M. R. Amieva, Stanford University, USA.

the gland-associated populations induce inflammation and hyperplasia of the gastric tissue and are critical for persistence and competition, understanding how $H$. pylori establishes and maintains this chronic reservoir in the glands will improve $H$. pylori clearance strategies to prevent pathology and disease," he concludes.

Katrina Ray

persist in the stomach 5
ORIGINAL ARTICLE Fung, C. et al. Highresolution mapping in the gastric glands control Helicobacter pylori colonization of the stomach. PLoS Biol. 17, e3000231 (2019)

\title{
GUT MICROBIOTA
}

\section{Anaerobic intestine-on-a-chip system enables complex microbiota co-culture}

A new experimental anaerobic microfluidic intestine-on-a-chip system has been developed that includes control of physiologically relevant oxygen gradients. This system enabled sustained co-culture of living human intestinal epithelium with a complex microbiota and led to improved intestinal barrier function compared with aerobic conditions.

Donald Ingber and colleagues have been developing organ chip technology for over 10 years. After noticing obstacles in the co-culture of microbial and human cells, they turned their expertise to developing a system that could recapitulate the dynamic host-microbiota interface.

The authors had previously described a two-channel microfluidic device lined with human intestinal cells that enabled stable co-culture of mucus-producing epithelium with human gut microbiota. However, culturing a fully typical human gut microbiota, including aerobic and anaerobic bacteria, required establishing the physiologically relevant oxygen gradients that are found in the large intestine.

"We leveraged our organ chip technology to recreate an oxygen gradient across a living human endothelial-epithelial interface that we reconstructed in vitro inside the chip," explains Ingber. The previous device was modified to include microscale oxygen sensors and then the chips, lined by $\mathrm{Caco} 2$ cells or human organoidderived epithelial cells, were housed in an anaerobic chamber. A complex microbiota community derived from human stool samples was then added. "This system enabled us to keep $>200$ different human commensal aerobic and anaerobic microorganisms alive in direct contact with patient-derived human intestinal epithelium for at least

5 days," reports Ingber. "This has never been done before."

In comparisons with aerobic chips, microbial diversity was found to be substantially improved with the anaerobic chips and the range of species abundances were in line with those reported by the Human Microbiome Project. The researchers also examined intestinal barrier function by quantifying permeability. "The integrity of the intestinal barrier was actually better under these more in vivo-like conditions with a hypoxia gradient," says Ingber.

Through improved modelling of the human intestine, this tool could facilitate discovery and development of microbiome-related therapeutics. "We are now using this system to model the contributions of the microbiome to malnutrition and to develop therapeutics to ameliorate this disease," concludes Ingber. "We also are integrating immune cells into the model."

lain Dickson

related therapeutics
ORIGINAL ARTICLE Jalili-Firoozinezhad, S. et al. A complex human gut microbiome cultured in an anaerobic intestine-on-a-chip. Nat. Biomed. Eng. https://doi.org/10.1038/s41551-019-0397-0 (2019) 